\title{
Evolutionary Artificial Neural Networks as Tools for Predicting the Internal Structure of Microemulsions
}

\author{
F. Podlogar, R. Šibanc, M. Gašperlin \\ University of Ljubljana, Faculty of Pharmacy, 1000 Ljubljana, Aškerčeva 7, SI-Slovenia \\ Received, February 11, 2008; Revised, March 9, 2008; Accepted, March 11, 2008; Published March 16, 2008.
}

\begin{abstract}
PURPOSE. The purpose of this study was to predict microemulsion structures by creating two artificial evolutionary neural networks (ANN) combined with a genetic algorithm. The first ANN would be able to determine the type of microemulsion from the desired composition, and the second to determine the type of microemulsion directly from a differential scanning calorimetry (DSC) curve. METHODS. The algorithms and the structures for each ANN were constructed and programmed in $\mathrm{C}++$ computer language. The ANNs had a feed forward structure with one hidden level and were trained using a genetic algorithm. DSC was used to determine the microemulsion type. RESULTS. The ANNs showed very encouraging accuracy in predicting the microemulsion type from its composition and also directly from the DSC curve. The percentage success, calculated over the tested data, was over $90 \%$. This enabled us, with satisfactory accuracy, to construct several pseudoternary diagrams that could facilitate the selection of the microemulsion composition to obtain the optimal desired drug carrier. CONCLUSIONS. The ANN constructed here, enhanced with a genetic algorithm, is an effective tool for predicting the type of microemulsion. These findings provide the basis for reducing research time and development cost for characterizing microemulsion properties. Its application would stimulate the further development of such colloidal drug delivery systems, exploit their advantages and, to a certain extent, avoid their disadvantages.
\end{abstract}

\section{INTRODUCTION}

Microemulsions (ME) are dispersions of oil in water, or vice versa, stabilized with a surface active film composed of surfactant and often also cosurfactant. They are well known colloidal drug delivery systems, because of their spontaneous formation, optical transparency and thermodynamic stability. They have the ability to improve bioavailability and/or to control the release of the incorporated drug $(1,2)$.

To find the optimal microemulsion composition for a desired drug release profile it is almost imperative to be able to characterize the microemulsion type and structure, which, in turn, depend on the ratio of the selected components. As it might seem futile to determine the structure of a liquid systems, the thermodynamic properties of microemulsion assure that oil and water phase, separated by the surfactants form a variety of different internal metastable formations, from simple droplets of oil in water (and vice versa) to the large bicontinuous formations and lamellar phases. Although thermodynamic properties enable the transitions among structures they remain relatively stable over a prolonged period of time. Still, their determination is far from trivial matter $(3,4)$. There are several methods available for analyzing the structure of microemulsions, such as differential scanning calorimetry (DSC), small angle X-ray scattering (SAXS), conductivity and surface tension measurement, photon correlation spectroscopy (PCS), transmission electron microscopy (TEM) and rheology (5-13). However most of them are very time consuming. Since pharmaceutically applicable microemulsions usually consist of at least four components, the classical trial-error approach for realizing the desired properties is costly and does not guarantee success. Even with the extensive and detailed studies there would still be possible to overlook potentially the best solution. The interactions between the components are too complex at the molecular level to allow construction of a mathematical function that would enable accurate prediction of the microemulsion properties.

Corresponding Author: Prof. Dr. Mirjana Gašperlin Faculty of Pharmacy, 1000 Ljubljana, Aškerčeva 7, SISlovenia E-mail address: mirjana.gasperlin@ffa.uni-lj.si 
However, a nonlinear mathematical approach, such as artificial neural networks $(14,15)$, constitutes a possible approach, especially if combined with a genetic algorithm. In the field of microemulsions an important research was done by Richardson et al. where they showed that back propagation ANN could be successfully used for predicting phase behaviour of theirs microemulsions (16).

Artificial neural networks (ANNs) are computational models of the human brain. They contain fully interconnected process units called artificial neurons $(14,17,18)$. Similar to the brain, the ANN receives input data, which are then processed by activation of neurons, and the network yields output. There are several characteristics that define the topology of the network structure and learning algorithms. ANNs can contain several hidden layers or even none. They can be recurrent or non-recurrent, both of which define the direction of the output. They could have a supervised or unsupervised form of learning. Input values may be binary or continuous. For the activation function of the neurons, linear, step, sigmoid or other functions can be used. The combination of these characteristics gives rise to several distinct types of ANN. Depending on the situation each could be more advantageous in terms of producing better results for the specific problem.

The majority of ANNs including the model of Richardson et al. (16) use a back propagation algorithm of learning. It is also possible to introduce a genetic algorithm to the classic feed forward ANN to form an evolutionary neural network (23). A genetic algorithm is a computer optimization technique that uses models inspired by evolutionary biology such as mutation, natural selection, and recombination. Similar to their natural counterparts, artificial chromosomes are used to encode the solution of a trained neural network (19). These chromosomes are subjected to the following basic procedures: the selection of solutions (chromosomes) based on their fitness, their reproduction by crossover of genes, and mutation for random change of genes (14). The main control parameters that define the process are the number of the initial generation (and size of the mating pool), the fitness function, which is a measure that can be used to compare solutions which is better, the selection method for breeding, the crossover probability which randomly determines whether crossover takes place, and the probability of mutation $(14,20)$. Also, it is very common to carry out a training exercise several times, with different initial populations, when applying genetic algorithms in order to obtain the best, optimal and global, rather than local, solutions.

The aim of the present study has been to create two artificial evolutionary neural networks (ANN), combined with a genetic algorithm, for predicting microemulsion structures. The first ANN would be able to determine the type of microemulsion from the desired composition, and the second to determine the type of microemulsion directly from a DSC curve. This is possible since DSC can enable the type of microemulsion to be determined by measuring the freezing peak of the water. Microemulsions were prepared with pharmaceutically acceptable components. Samples were analyzed with DSC and inspected visually to obtain information about their type and general structure. These data were then used to train both ANNs. Similar work for predicting microemulsion structure using ANN was done by Richardson et al (16). Their study was focused on ability of ANN to find potential cosurfactant for lecithin while our purpose was to develop ANN as a tool for predicting microstructures on the basis of experimental data for a given system. In addition we expect that ANN would be able to accelerate the interpretation of DSC data for elucidating microstructure.

\section{MATERIALS AND METHODS}

\section{Materials}

Isopropyl myristate (IPM) was obtained from Fluka Chemie $\mathrm{GmbH}$, Switzerland, and used as the lipophilic phase of microemulsions. Tween $40^{\circledR}$ (TW40) - polyoxyethylene (20) sorbitan monopalmitate (Fluka chemie $\mathrm{GmbH}$, Switzerland) was used as surfactant and Imwitor ${ }^{\mathbb{B}}$ 308 (IMW) - glyceryl caprylate (Condea Chemie $\mathrm{GmbH}$, Germany) as cosurfactant. Twice distilled water was used as the hydrophilic phase.

\section{Structure determination of microemulsions with differential scanning calorimetry}

A differential scanning calorimeter DSC-4 (Perkin Elmer, USA) was used, with a nitrogen flow of $20 \mathrm{ml} / \mathrm{min}$ as purge gas. Approximately 5 to $15 \mathrm{mg}$ of sample was weighed precisely into a small aluminium pan and quickly sealed hermetically to prevent water evaporation. The 
empty sealed pan was used as reference. Samples were cooled from $30^{\circ} \mathrm{C}$ to $-60^{\circ} \mathrm{C}$ (cooling rate 10 $\mathrm{K} / \mathrm{min}$ ).

\section{Artificial neural network structure and training}

\section{Structure of the ANN used to determine the type of microemulsion from its composition}

To determine the type of microemulsion from its composition we programmed a feed-forward network, with the final architecture involving 4 input neurons, a single hidden layer of 12 neurons, and 5 output neurons. Preliminary training runs were performed using hidden layers involving 3 to 18 neurons. Significant improvements in prediction were achieved up to a hidden layer size of 10; prolonged learning time led to no greater accuracy and diminishing returns were obtained with networks featuring more than 15 hidden neurons. We experimented also with ANNs containing two hidden neuron layers with up to 20 hidden neurons in total, however the results were not satisfactory. For the activation function, a sigmoid function ranging from 0 to 1 was used. The weights of the ANN were determined by the genetic algorithm as explained bellow.

The 4 input neurons corresponded to the four components used to produce the microemulsion: Tween (surfactant), Imwitor (cosurfactant), IPM (lipophilic phase) and water (hydrophilic phase). The raw weight $\%$ for each component was first submitted to a mathematical transformation. Each of them was subtracted by the average value of all weight \% of the same component (of all the samples in the training set) and divided by their standard deviation value (see Eq. 1). This mathematical transformation provides better learning of the ANN (21).

$$
p=\frac{x_{i}-A V G}{S D}
$$

where $x_{i}$ is the weight $\%, A V G$ is the average value of weight ratios and $S D$ their standard deviation.

Each of the 5 output neurons represents one possible structure - $\mathrm{O} / \mathrm{W}$ microemulsion, bicontinuous microemulsion, $\quad \mathrm{W} / \mathrm{O}$ microemulsion, $\mathrm{O} / \mathrm{W}$ emulsion or $\mathrm{W} / \mathrm{O}$ emulsion. The output value of each output neuron represents the probability of the microemulsion being a certain type for the sample in question. The winning solution was the one whose output neuron had the highest probability. We used a supervised form of learning, which was discontinued after the classification error dropped below $1 \%$.

\section{The structure of the ANN used to determine the type of microemulsion from its DSC curve}

To determine the type of microemulsion from its DSC curve directly, a second feed-forward ANN with 1 hidden layer was constructed containing 100 input neurons (i.e. the input data of the DSC curve), a single layer of 5 hidden neurons (the network was otherwise tested with 3-200 hidden neurons) and 5 output neurons. All computational and training procedures were as described bellow.

\section{Training the ANN using a genetic algorithm}

A genetic algorithm was used to determine the weight (genes) values as W1 1, W1 2, W1 3, up to W4 12. Each weight is represented as a gene in the chromosome (solution); the first number represents the first neuron (for example the input neuron) and the second number represents the second neuron (for example in hidden neuron).

The initial population consisted of 50 different chromosomes where each represents a certain weight combination. Larger populations did not give better solution, but only prolonged the computation. The fitness function to determine which solution is better was the average squared error (see Eq. 2).

$$
A S E=\sum_{i=1}^{n} \frac{\left(t_{i}-\sigma_{i}\right)^{2}}{n}
$$

where, ASE is average square error, $t_{i}$ actual value, $\sigma_{i}$ target value and $\mathrm{n}$ total number of the data.

The best solution, in this case a combination of weights, is the one that yields the lowest average square error. To obtain the offspring population, the top five solutions were automatically copied. A tournament ranking system was then implemented, in which the winning chromosome, from 4 randomly selected chromosomes, with the best fitness was copied and then subjected to 
crossover or mutation and transferred to the offspring population. This type of selection leads to the best solutions but still allows the chance that a minor solution could contribute, which might prove useful in the following generations. We constructed two crossover and ten mutation models. Each model had an equal chance of being implemented on the designated chromosome. Crossover was implemented by random crossing of the part of the chromosome between two parent chromosomes to yield two new offspring chromosomes, or by their linear combination. The mutation models usually employed the addition of a small value to a specific gene (weight value) or its multiplication by a random factor. The algorithms and the structures were constructed for each $\mathrm{ANN}$ and programmed in $\mathrm{C}++$ computer language.

\section{RESULTS AND DISCUSSION}

This study follows on from the research $(3,4)$, where we determined the exact structure and type of pharmaceutically applicable microemulsions composed of the same components as in this study. For this purpose, components (surfactant, cosurfactant, oil and water) suitable for dermal application were selected and pseudoternary diagrams were constructed where the area of microemulsion formation was presented. The ratio of surfactant to cosurfactant was fixed. In this way, the results could be presented in two dimensions. Preliminary studies showed that the most appropriate surfactant to cosurfactant ratio was $1: 1$, where microemulsions were formed if more than $30 \mathrm{wt} . \%$ of surfactant was present. The least amount of surfactant is desirable to avoid irritation of the skin as much as possible. However, with lower surfactant concentrations it is often hard to obtain stable microemulsion systems. In addition to the most appropriate pseudoternary diagram, with the surfactant to cosurfactant ratio of $1: 1$, we prepared two additional diagrams with the ratio fixed at 2:1 and 1:2. In total, 170 samples (from all three diagrams) were used to train the network. An example is shown in Figure1, which represents the training set samples on the pseudoternary diagram for a surfactant to cosurfactant ratio of $1: 1$.

\section{Obtaining the training set data}

The microemulsion samples were inspected visually and analyzed by DSC. By visual inspection, microemulsions, which were clear and transparently dispersed were distinguished from classical milky emulsions. Using DSC, it is possible to determine the state of water in the microemulsion system and consequently the structure. In most cases the distinction is made between bulk and bound water $(5,6,7)$. The cooling curves of the microemulsion samples were analyzed in our previous work $(3,4)$. For the $\mathrm{W} / \mathrm{O}$ microemulsions, the largest peak appeared at approx. $-8^{\circ} \mathrm{C}$, indicating solidification of IPM (lipophilic phase). The second, much smaller but important peak at approximately $-42^{\circ} \mathrm{C}$, indicates freezing of surfactant and cosurfactant mixture. For these systems is significant that water is interacting strongly with other components and no freezing of water molecules is detected. When analysing the bicontinuous type of microemulsion we observed that water molecules have fewer interactions with surfactant molecules and freezing of "bound" water in the system is seen as a new peak at approximately $-50^{\circ} \mathrm{C}$. In the $\mathrm{O} / \mathrm{W}$ type of microemulsion a large sharp peak appears at approximately $-17^{\circ} \mathrm{C}$, which indicates, freezing of supercooled water with fewer interactions (22). This provides the key to understanding how it is possible to distinguish between different microemulsion types using DSC.

\section{Determining the type of microemulsion from its composition}

When the ANN was trained (accuracy of predictions on training data above 99\%), we instructed the network to predict the structures for all possible composition combinations for the pseudoternary diagrams with the surfactant to cosurfactant ratios of 1:1, 2:1, 1:2 and 1.5:1. The latter diagram was constructed to study how well would ANN be able to predict the type of microemulsion for the unknown surfactant to cosurfactant ratio, away from the actual training set. The results are presented in Figure 2. In order to assess the validity of the network approach, microemulsions previously not tested were selected (all types of microemulsion were covered). They were inspected visually, analyzed by DSC and the results were compared with the ANN prediction. The percentage success calculated over the tested data was $90 \%$ (Table 1), which was very encouraging. Constructing this kind of ANN, combined with a genetic algorithm, shows the possibility of predicting the microemulsion type for previously untested compositions. 


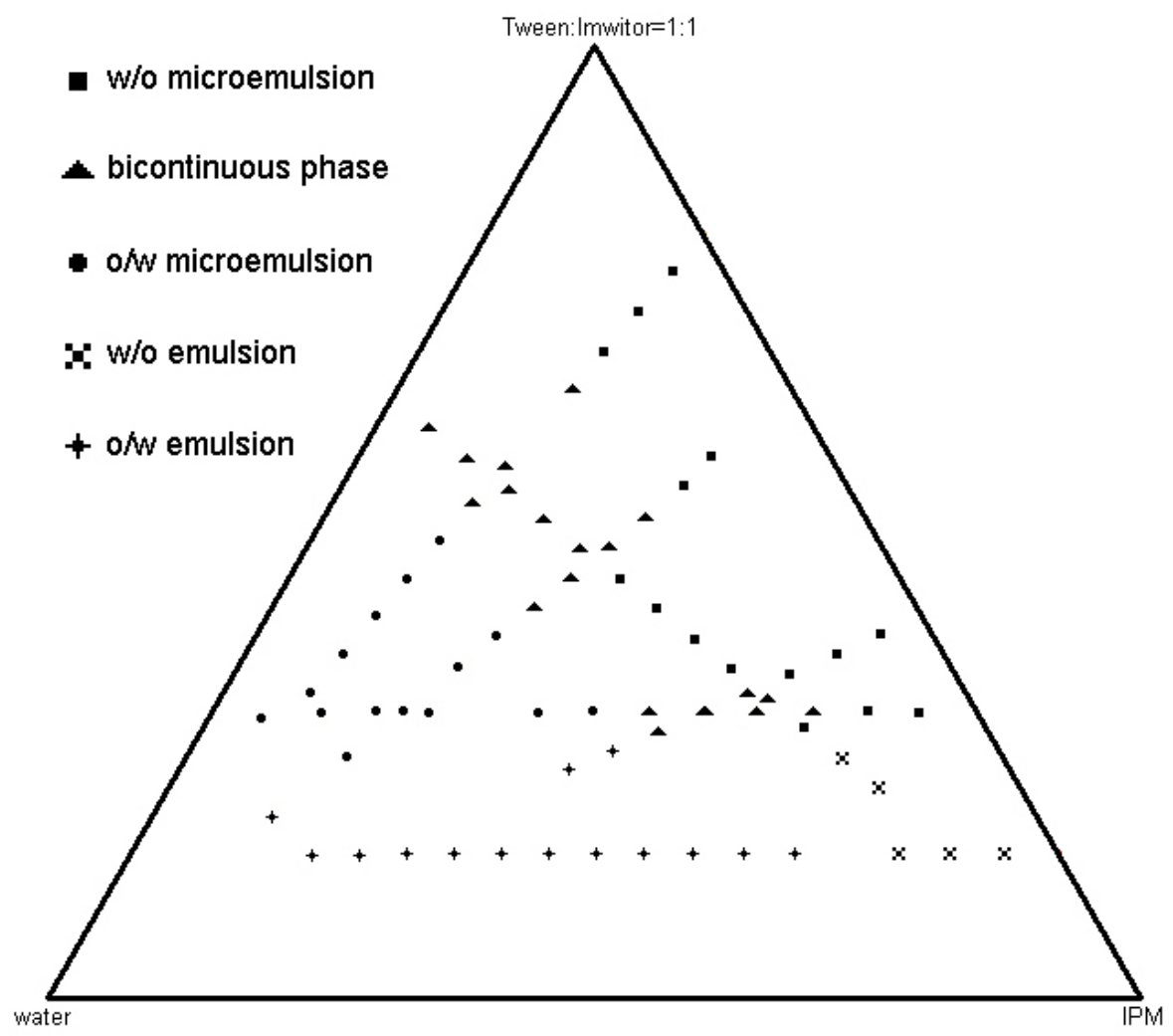

Figure1: An example of the training set on the pseudoternary diagram where surfactant to cosurfactant ratio is fixed to $1: 1$.

The general limitations still apply since the accuracy of prediction diminishes if the composition of the microemulsion in question is significantly different from the actual training set. The described ANN can be used only to predict accurately the construction of the phase diagram for four component microemulsions and with great accuracy if the surfactant to cosurfactant ratio is from $1: 2$ to $2: 1$. However, there is no reason that the same principles could not be used to construct a similar network where the training data would involve other surfactants, oils and different surfactant to cosurfactant ratios, and it is unlikely that these results would be less successful. In general, the information acquired may be quite useful, not only because predicting the type would minimize the time and cost of microemulsion characterization, but would also be helpful in predicting the drug release depending on the composition (4). This would be achieved far more easily and with less effort than by using the classical trial-error approach.

\section{Determining the type of microemulsion from DSC curve directly}

If we are able to predict the microemulsion type by analyzing DSC data, we should be able to design also ANN that would be able to predict the type of microemulsion from DSC curve directly. For this purpose we constructed another ANN. For the training set we used several DSC curves of the samples with surfactant to cosurfactant ratio of 1:1. Examples of the typical curves used to train the network are presented in Figure 3.

After completing the network learning cycles, we selected several curves, not involved previously in the learning process of the ANN that related to several different types of microemulsion, in order to test the accuracy of network prediction. The results show 90\% accuracy in prediction (Table 2), which is very encouraging. The implications of these findings could be very important. With the advent of autosamplers attached DSC, several samples can now be prepared and analyzed automatically. Not only the raw results (thermograms) but also their interpretation would be available in terms of the microemulsion type and structure. 

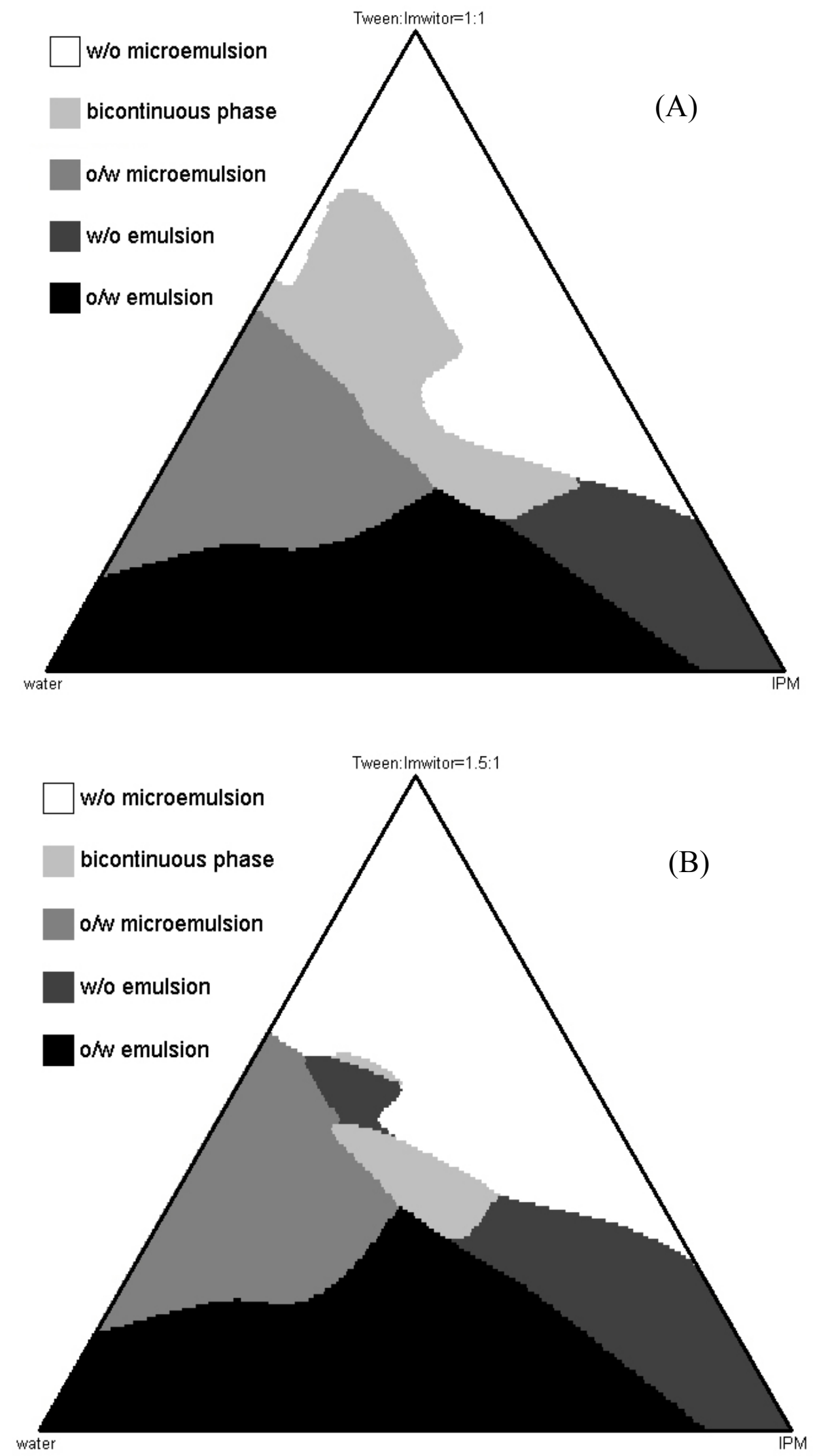
Figure 2. Continued....
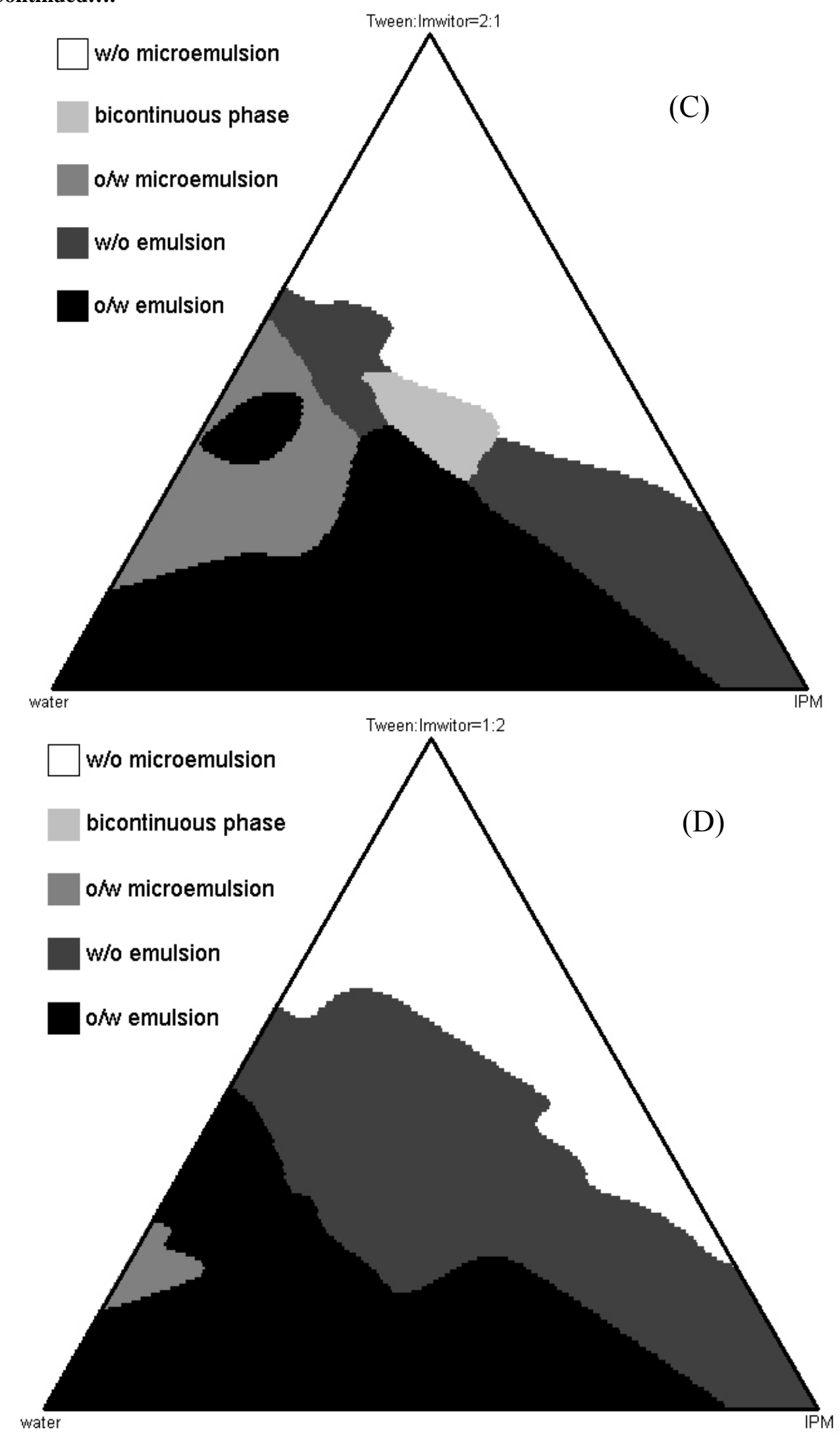

Figure 2: Solutions, predicted by ANN. The respective surfactant to cosurfactant ratios are: a) $1: 1$, b) $1,5: 1$, c) $2: 1$, d) $1: 2$, respectively. There were no training points on the diagram with $1,5: 1$ ratio. 
Table 1. Validation of an artificial neural network for its ability to determine the type of microemulsion from its composition using randomly selected, previously untested samples.

\begin{tabular}{cccccc}
\hline $\begin{array}{c}\text { Tween } \\
\text { wt. ratio }\end{array}$ & $\begin{array}{c}\text { Imwitor } \\
\text { wt. ratio }\end{array}$ & $\begin{array}{c}\text { IPM } \\
\text { wt. ratio }\end{array}$ & $\begin{array}{c}\text { water } \\
\text { wt. ratio }\end{array}$ & $\begin{array}{c}\text { predicted ME } \\
\text { type by ANN }\end{array}$ & $\begin{array}{c}\text { actual ME } \\
\text { type }\end{array}$ \\
\hline 0.1347 & 0.1347 & 0.0700 & 0.6606 & $\mathrm{O} / \mathrm{W}$ & $\mathrm{O} / \mathrm{W}$ \\
0.2116 & 0.2116 & 0.1651 & 0.4117 & $\mathrm{O} / \mathrm{W}$ & $\mathrm{O} / \mathrm{W}$ \\
0.1882 & 0.1882 & 0.5500 & 0.0736 & $\mathrm{~W} / \mathrm{O}$ & $\mathrm{W} / \mathrm{O}$ \\
0.1936 & 0.1936 & 0.0683 & 0.5445 & $\mathrm{O} / \mathrm{W}$ & $\mathrm{O} / \mathrm{W}$ \\
0.2786 & 0.2786 & 0.2351 & 0.2078 & bicontinuous & bicontinuous \\
0.1680 & 0.1680 & 0.4206 & 0.2434 & bicontinuous & bicontinuous \\
0.2084 & 0.2084 & 0.0785 & 0.5048 & $\mathrm{O} / \mathrm{W}$ & $\mathrm{O} / \mathrm{W}$ \\
0.2705 & 0.2705 & 0.0842 & 0.3749 & bicontinuous & bicontinuous \\
0.1906 & 0.1906 & 0.3312 & 0.2877 & bicontinuous & bicontinuous \\
0.1494 & 0.1494 & 0.4897 & 0.2115 & bicontinuous & bicontinuous \\
0.2645 & 0.2645 & 0.2190 & 0.2520 & bicontinuous & bicontinuous \\
0.3652 & 0.2435 & 0.1929 & 0.1984 & $\mathrm{~W} / \mathrm{O}$ & $\mathrm{W} / \mathrm{O}$ \\
0.4605 & 0.3070 & 0.0522 & 0.1802 & $\mathrm{~W} / \mathrm{O}$ & $\mathrm{W} / \mathrm{O}$ \\
0.4639 & 0.3093 & 0.1803 & 0.0464 & $\mathrm{~W} / \mathrm{O}$ & $\mathrm{W} / \mathrm{O}$ \\
0.2849 & 0.1900 & 0.3108 & 0.2143 & $\mathrm{~W} / \mathrm{O}$ & $\mathrm{W} / \mathrm{O}$ \\
0.2519 & 0.1680 & 0.1130 & 0.4671 & $\mathrm{O} / \mathrm{W}$ & $\mathrm{O} / \mathrm{W}$ \\
0.3151 & 0.2101 & 0.3941 & 0.0808 & $\mathrm{~W} / \mathrm{O}$ & $\mathrm{W} / \mathrm{O}$ \\
0.1858 & 0.1858 & 0.2877 & 0.3408 & bicontinuous & $\mathrm{O} / \mathrm{W}$ \\
0.2693 & 0.2693 & 0.0525 & 0.4090 & bicontinuous & $\mathrm{O} / \mathrm{W}$ \\
0.1525 & 0.1525 & 0.0562 & 0.6388 & $\mathrm{O} / \mathrm{W}$ & $\mathrm{O} / \mathrm{W}$ \\
\hline
\end{tabular}

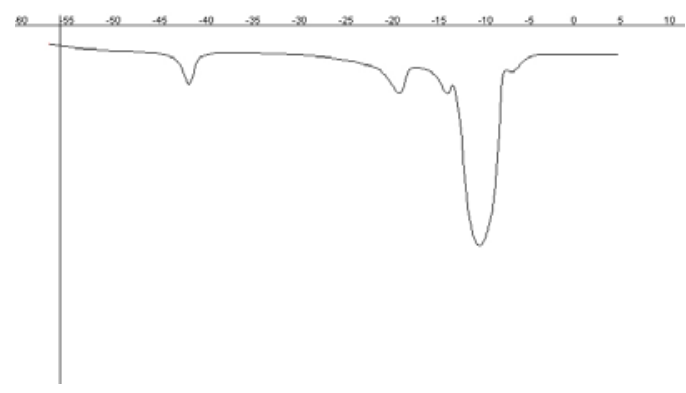

(a)

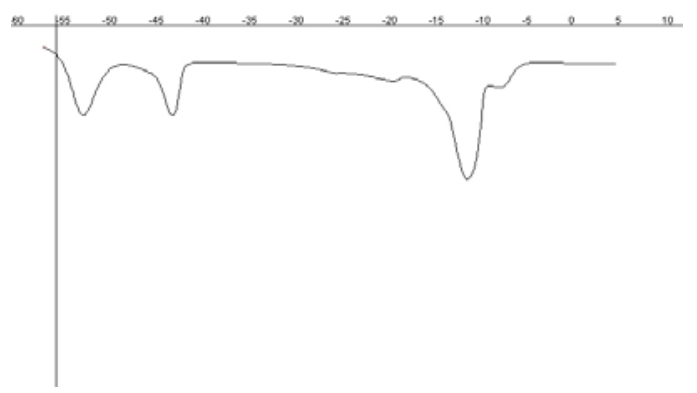

(b)

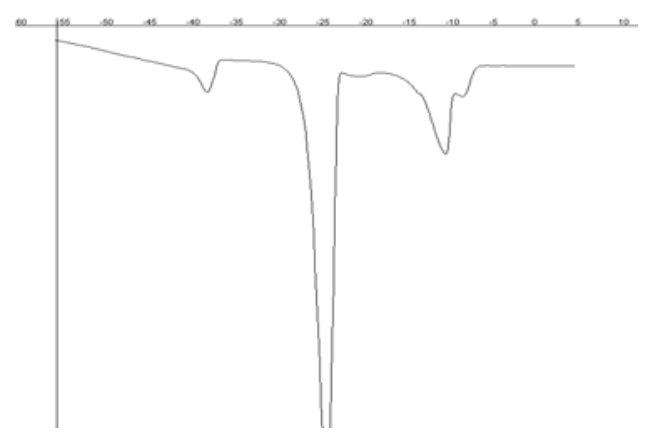

(c)

Figure 3: An example of typical DSC curves (thermograms) that were used to train the network so that would be capable to predict the type of microemulsion from DSC curve directly. a) W/O microemulsion, b) bicontinuous phase, c) $\mathrm{O} / \mathrm{W}$ microemulsion. 
Table 2. Validation of an artificial neural network for its ability to determine the type of microemulsion directly from its DSC curve using randomly selected, previously untested samples.

\begin{tabular}{cccccc}
\hline $\begin{array}{c}\text { Tween } \\
\text { wt. ratio }\end{array}$ & $\begin{array}{c}\text { Imwitor } \\
\text { wt. ratio }\end{array}$ & $\begin{array}{c}\text { IPM wt. } \\
\text { ratio }\end{array}$ & $\begin{array}{c}\text { water wt. } \\
\text { ratio }\end{array}$ & $\begin{array}{c}\text { predicted ME type by } \\
\text { ANN }\end{array}$ & actual ME type \\
\hline 0.2839 & 0.2839 & 0.3315 & 0.1007 & $\mathrm{~W} / \mathrm{O}$ & $\mathrm{W} / \mathrm{O}$ \\
0.2524 & 0.2524 & 0.2944 & 0.2008 & bicontinuous & bicontinuous \\
0.2208 & 0.2208 & 0.2580 & 0.3005 & bicontinuous & bicontinuous \\
0.2053 & 0.2053 & 0.2397 & 0.3498 & bicontinuous & bicontinuous \\
0.1895 & 0.1895 & 0.2211 & 0.4000 & $\mathrm{O} / \mathrm{W}$ & $\mathrm{O} / \mathrm{W}$ \\
0.2684 & 0.2684 & 0.3130 & 0.1503 & $\mathrm{~W} / \mathrm{O}$ & $\mathrm{W} / \mathrm{O}$ \\
0.2367 & 0.2367 & 0.2764 & 0.2502 & bicontinuous & $\mathrm{bicontinuous}$ \\
0.1263 & 0.1263 & 0.1474 & 0.6000 & $\mathrm{O} / \mathrm{W}$ & $\mathrm{O} / \mathrm{W}$ \\
0.1736 & 0.1736 & 0.2027 & 0.4501 & $\mathrm{~W} / \mathrm{O}$ & $\mathrm{O} / \mathrm{W}$ \\
0.2839 & 0.2839 & 0.1001 & 0.3321 & bicontinuous & bicontinuous \\
0.2686 & 0.2686 & 0.1497 & 0.3132 & bicontinuous & bicontinuous \\
0.2526 & 0.2526 & 0.1998 & 0.2950 & bicontinuous & bicontinuous \\
0.2369 & 0.2369 & 0.2498 & 0.2765 & bicontinuous & bicontinuous \\
0.2213 & 0.2213 & 0.2999 & 0.2575 & $\mathrm{~W} / \mathrm{O}$ & $\mathrm{W} / \mathrm{O}$ \\
0.1891 & 0.1891 & 0.4005 & 0.2213 & $\mathrm{~W} / \mathrm{O}$ & $\mathrm{W} / \mathrm{O}$ \\
0.1736 & 0.1736 & 0.4503 & 0.2025 & $\mathrm{~W} / \mathrm{O}$ & $\mathrm{W} / \mathrm{O}$ \\
0.1502 & 0.1502 & 0.5248 & 0.1747 & $\mathrm{~W} / \mathrm{O}$ & $\mathrm{W} / \mathrm{O}$ \\
0.1495 & 0.1495 & 0.6471 & 0.0539 & $\mathrm{~W} / \mathrm{O}$ & $\mathrm{W} / \mathrm{O}$ \\
0.1502 & 0.1502 & 0.5996 & 0.1001 & $\mathrm{~W} / \mathrm{O}$ & $\mathrm{W} / \mathrm{O}$ \\
0.1508 & 0.1508 & 0.4973 & 0.2012 & bicontinuous & $\mathrm{b}$ \\
0.1499 & 0.1499 & 0.2998 & 0.4005 & $\mathrm{O} / \mathrm{W}$ & $\mathrm{O} / \mathrm{W}$ \\
0.1495 & 0.1495 & 0.1016 & 0.5994 & $\mathrm{O} / \mathrm{W}$ & $\mathrm{O} / \mathrm{W}$ \\
0.1504 & 0.1504 & 0.1500 & 0.5492 & bicontinuous & $\mathrm{O} / \mathrm{W}$ \\
0.1464 & 0.1464 & 0.0485 & 0.6587 & $\mathrm{O} / \mathrm{W}$ & $\mathrm{O} / \mathrm{W}$ \\
0.1502 & 0.1502 & 0.4496 & 0.2501 & bicontinuous & $\mathrm{bicontinuous}$ \\
0.1506 & 0.1506 & 0.3995 & 0.2994 & bicontinuous & $\mathrm{bicontinuous}$ \\
0.2998 & 0.2998 & 0.0502 & 0.3502 & bicontinuous & $\mathrm{bicontinuous}$ \\
0.2996 & 0.2996 & 0.1008 & 0.3001 & bicontinuous & $\mathrm{bicontinuous}$ \\
0.3001 & 0.3001 & 0.1500 & 0.2498 & bicontinuous & bicontinuous \\
0.2998 & 0.2998 & 0.2000 & 0.2004 & $\mathrm{~W} / \mathrm{O}$ & $\mathrm{W} / \mathrm{O}$ \\
0.3000 & 0.3000 & 0.2499 & 0.1501 & bicontinuous & $\mathrm{W} / \mathrm{O}$ \\
0.2998 & 0.2998 & 0.3001 & 0.1004 & $\mathrm{~W} / \mathrm{O}$ & $\mathrm{W} / \mathrm{O}$ \\
0.3002 & 0.3002 & 0.3497 & 0.0499 & $\mathrm{~W} / \mathrm{O}$ & $\mathrm{W} / \mathrm{O}$ \\
\hline & & & &
\end{tabular}

\section{Future implications}

The next step would be to implement other complementary methods, such as conductivity, surface tension measurements and SAXS, into ANN for microemulsion analysis in order to correlate data regarding type and structure directly to drug release. Due to the nature of several nonlinear equations that are often unknown and are dependent on the system, it is almost impossible to calculate the drug release directly, however using ANNs, enhanced with a genetic algorithm, such predictions could be possible. Since the microemulsions are formed spontaneously, the critical step in the formulation development is to select the proper pharmaceutically applicable components, which are able to form microemulsions. After preparation and sample analysis the results obtained would be automatically used to train ANN. It would then be possible to select different types of microemulsions and determine separately the drug release or permeation. The findings could then be inserted into ANN, to give a powerful tool, capable of predicting biopharmaceutical properties of the systems with 
the desired drug. We believe this approach could vastly improve the development of potentially more suitable microemulsion carrier systems.

\section{CONCLUSION}

We constructed two evolutionary ANNs enhanced with a genetic algorithm, one being able to predict the type of microemulsion from its composition and the other to predict the type of microemulsion directly from the DSC curve. Both show an encouraging accuracy of $90 \%$ in prediction. They provide the basis for reducing research time and development cost for the characterization of microemulsion properties and drug release ability as the drug release is dependant on the microemulsion properties (4). It would stimulate the development of such colloidal drug delivery systems to a greater extent, exploit their advantages and, to a certain extent, avoid their disadvantages.

\section{REFERENCES}

[1]. Tenjarla S. Microemulsions: An overview and pharmaceutical applications. Crit Rev Ther Drug, 16(5): 461-521, 1999.

[2]. Bagwe R. P., Kanicky J. R., Palla, B. J., et al. Improved drug delivery using microemulsions: Rationale, recent progress, and new horizonts. Crit Rev Ther Drug, 18(1): 77-140, 2001.

[3]. Podlogar F., Gašperlin M., Tomšič M., et al. Structural characterisation of water-Tween $40 \AA / I m w i t o r \quad 308 \circledR$-isopropyl myristate microemulsions using different experimental methods. Int J Pharm, 276(1-2): 115-128, 2004.

[4]. Podlogar F., Bešter-Rogač M. and Gašperlin M. The effect of internal structure of selected water - Tween $40^{\circledR}$ - Imwitor $308^{\circledR}$ - IPM microemulsions on ketoprofene release. Int $\mathrm{J}$ Pharm, 302(1-2): 68-77, 2005.

[5]. Garti N.; Thermal behavior of dispersed systems. Marcel Dekker, Inc, New York, pp 59181, 2001.

[6]. Schulz P. C. DSC analysis of the state of water in surfactant-based microstructures. J Therm Anal, 51: 135-149, 1998.

[7]. Erzahi S., Anserin A., Fanun M., et al. Subzero temperature behavior of water in microemulsions. Schulz P. C., Soltero J. F. A. and Puig, J. E. DSC analysis of surfactantbased microsystems. In: Thermal behaviour of dispersed systems. Marcel Dekker, Inc, New York, pp 59-181, 2001.

[8]. Glatter O., Orthaber D., Stradner A., et al. Sugar-Ester Nonionic Microemulsion: Structural Characterization. J Colloid and Interface Sci, 241: 215-225, 2001.
[9]. Choi S. M., Chen S.H., Sottman, T, et al. Measurement of interfacial curvatures in microemulsions using small-angle neutron scattering. Physica B, 241-243: 976-978, 1988.

[10]. Boned C., Pyrelasse J. and Saidi Z. Dynamic percolation of spheres in a continuum: The case of microemulsions. Phys Rev, E47: 468-478, 1993.

[11]. D'Aprano A., D'Arrigo G., Paparelli A., et al. Volumetric and Transport Properties of Water/AOT/n-Heptane Microemulsions. J Phys Chem, 97: 3614-3618, 1993.

[12]. Giustini M., Palazzo G., Colafemmina G., et al. Microstructure and Dynamics of the Water-inOil CTAB/n-Pentanol/n-Hexane/Water Microemulsion: A Spectroscopic and Conductivity Study. J Phys Chem, 70: 959-971, 1996.

[13]. Caron G. and Desnoyers J. E. Study of Ternary Systems Forming Stable Emulsions. J Colloid and Interface Sci, 119: 114-149, 1986.

[14]. Munakata T. Fundamentals of the new artificial intelligence. Springer, New York, 1998.

[15]. Richardson C. J. and Barlow D. J. Neural network computer simulation of medical aerosols. J Pharm Pharmacol, 48(6), 581-591, 1996.

[16]. Richardson C. J., Mbanefo A,. and Aboofazeli R., et al. Prediction of phase behavior in microemulsion systems using artificial neural networks. J Colloid and Interface Sci, 187: 296303, 1997.

[17]. Alany R.G., Agatonovic-Kustrin S., Rades T., et al. Use of artificial neural networks to predict quaternary phase systems from limited experimental data. J Pharmceut Biomed, 19: $443-452,1999$.

[18]. Erb L. J. Introduction to backwardation neural network computation. Pharm Res, 10 (2): 165170, 1993.

[19]. Davis L., Handbook of genetic algorithms, Van Nostrand Reinhold, New York, 1991.

[20]. Kim H. T., Kim B. Y., Park E. H., et al. Computer recognition of Alzheimer diseaseEEG using genetic algorithms and neural network. Future generation computer systems 21: 1124-1130, 2005.

[21]. $\mathrm{ftp}: / / \mathrm{rtfm} . \mathrm{mit} . \mathrm{edu} / \mathrm{pub} / \mathrm{usenet} / \mathrm{comp}_{\text {aai.neural- }}$ nets/comp.ai.neuralnets FAQ, Part 2 of 7: Learning; 19.5.2005

[22]. Milon J. J. G. and Braga S. L. Supercooling water in cylindrical capsules. $15^{\text {th }}$ symposium on Thermophysical properties, Boulder, Colorado, USA, june 22-27, 2003.

[23]. X. Yao, "Evolution of connectionist networks," in Preprints Int. Symp. AI, Reasoning and Creativity, T. Dartnall, Ed., Queensland, Australia. Griffith Univ., pp. 49-52, 1991. 